\title{
Battery-powered wireless monitoring system for geotechnical, hydrology and microseismic sensors using the MineHop mesh network
}

\author{
A Dulmage Mine Design Technologies Inc., Canada \\ N Ruddell Mine Design Technologies Australia, Australia
}

\begin{abstract}
Technology advances in recent years have made it possible for battery-powered wireless communication to thrive in underground mining environments. SMART geotechnical instruments from Mine Design Technologies (MDT), vibrating wire based instruments from many manufacturers, and most recently microseismic sensors embedded in the toe of MDT SMART instruments can now be monitored using the MineHop battery-powered wireless mesh network. This paper will discuss the different applications of the MineHop technology, the ease of installation and maintenance, specifications, and case studies including examples of typical installations.
\end{abstract}

\section{Introduction}

Some of the key constraints in deploying and commissioning sensor networks in an underground mining environment are that often the areas where sensors are to be deployed have limited access due to production traffic, and similarly due to high traffic hard wired sensor networks are inherently subject to damage by equipment, causing significant and ongoing maintenance issues.

With advancements in both battery technology, as well as ultra-low power radio frequency (RF) microchips, the idea of a battery-powered wireless sensor network for use in extremely rugged environments such as underground mining, has been realised. The small nature of the batteries, enclosures and antennae, allows the equipment to be deployed rapidly in areas with difficult access and pose little risk of damage by heavy equipment. Since the batteries used are of a high capacity, and the power consumption of the equipment is low, years of battery life can be achieved while maintaining real time monitoring of most types of geotechnical and hydrology instruments available today.

\section{System overview}

The system discussed in the paper is called the MineHop network, developed by Newtrax Technologies Inc. This network consists of three key components:

- Central server - located on surface to store sensor data in a secure database and act as a web server for user access and initiate email alarm notifications.

- Gateways - either Ethernet or Leaky Feeder based to provide a pathway between the underground RF network and the central server over the mine's existing network.

- Nodes - either repeater nodes for relaying signal to a gateway with connectivity to the mine network, or remote telemetry units (RTU) capable of reading and transmitting data from a wide variety of geotechnical and hydrology sensors.

An illustration of the network is presented in Figure 1. 


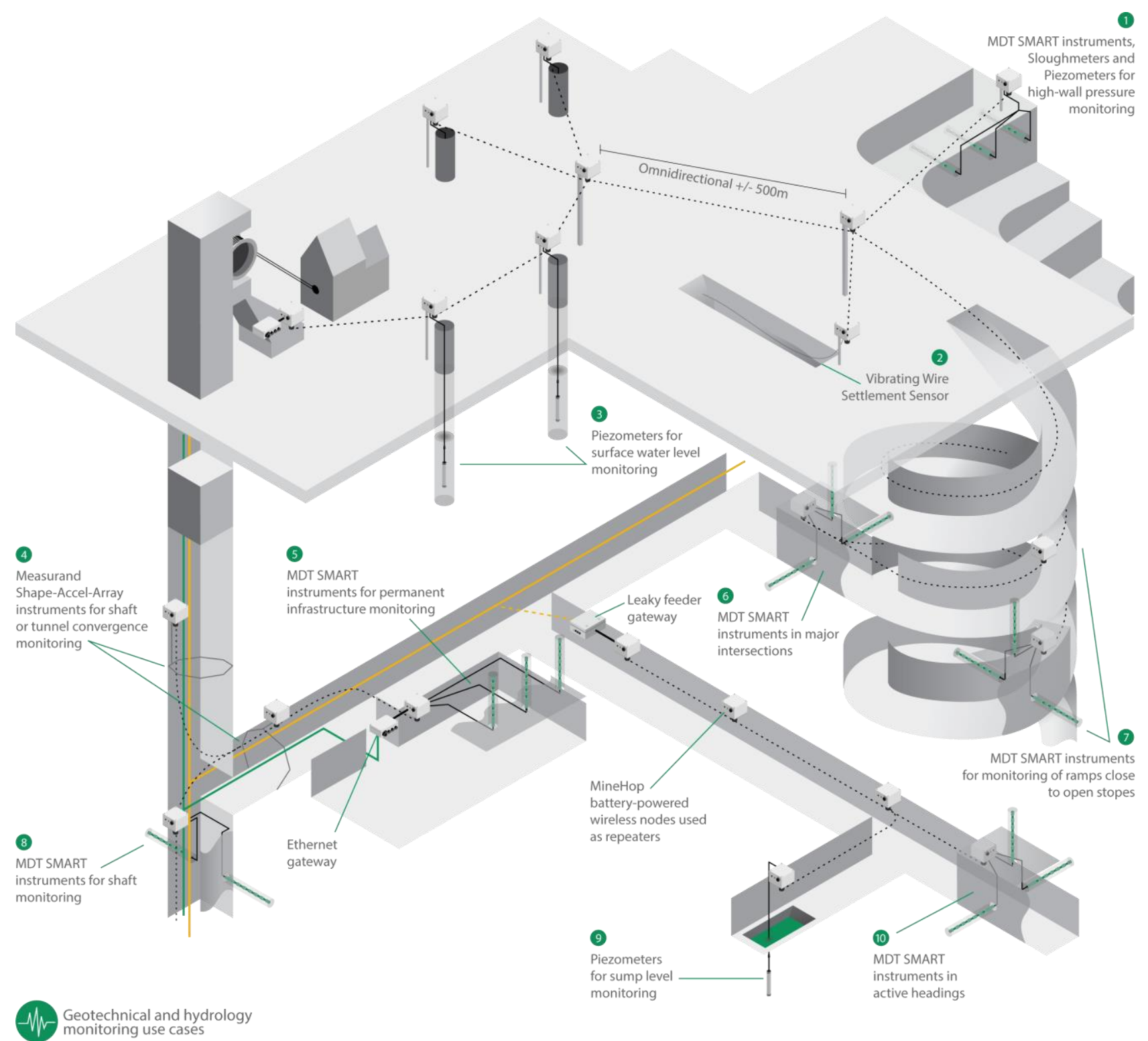

\section{Figure 1 Overview of network layout}

\subsection{Wireless nodes}

The key element in the battery powered RF network is the standard infrastructure node providing wireless network reach to sensor locations from a gateway access point, and can run on batteries for about three years - soon to be five years in the second half of 2015 . The RF chip is $902-928 \mathrm{MHz}$ and operates in the unlicensed $900 \mathrm{MHz}$ band, providing 50-150 m of typical range in an underground mining environment, or up to $600 \mathrm{~m}$ on surface. A standard infrastructure node is shown in Figure 2. 


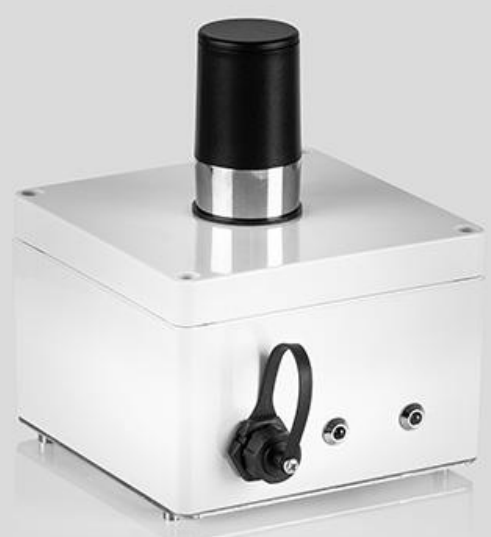

\section{Figure 2 Standard infrastructure node}

\subsection{Remote telemetry units}

For sensor interfacing, several versions of the standard infrastructure node have been developed, each with the same RF properties and typical three-year battery life as the standard infrastructure node. These include the MDT-RTU, VW-RTU and SAA-RTU, all designed to be relatively plug and play with the associated sensors.

\subsubsection{MDT-RTU}

These units are capable of reading analogue voltage inputs, as is found in SMART multi-point borehole extensometers (MPBX) and instrumented cable bolts for ground support monitoring, but can also include a variety of other potentiometer based sensors. The unit has been designed to accommodate up to three MPBX or cable bolts, equating to 18 analogue voltage inputs, often suiting a drive array with an instrument in each of the walls and one in the backs (roof). The unit also has on-board buffer storage to store readings over short periods if a connection with the wireless network is obstructed. An image of the MDT-RTU is shown in Figure 3.

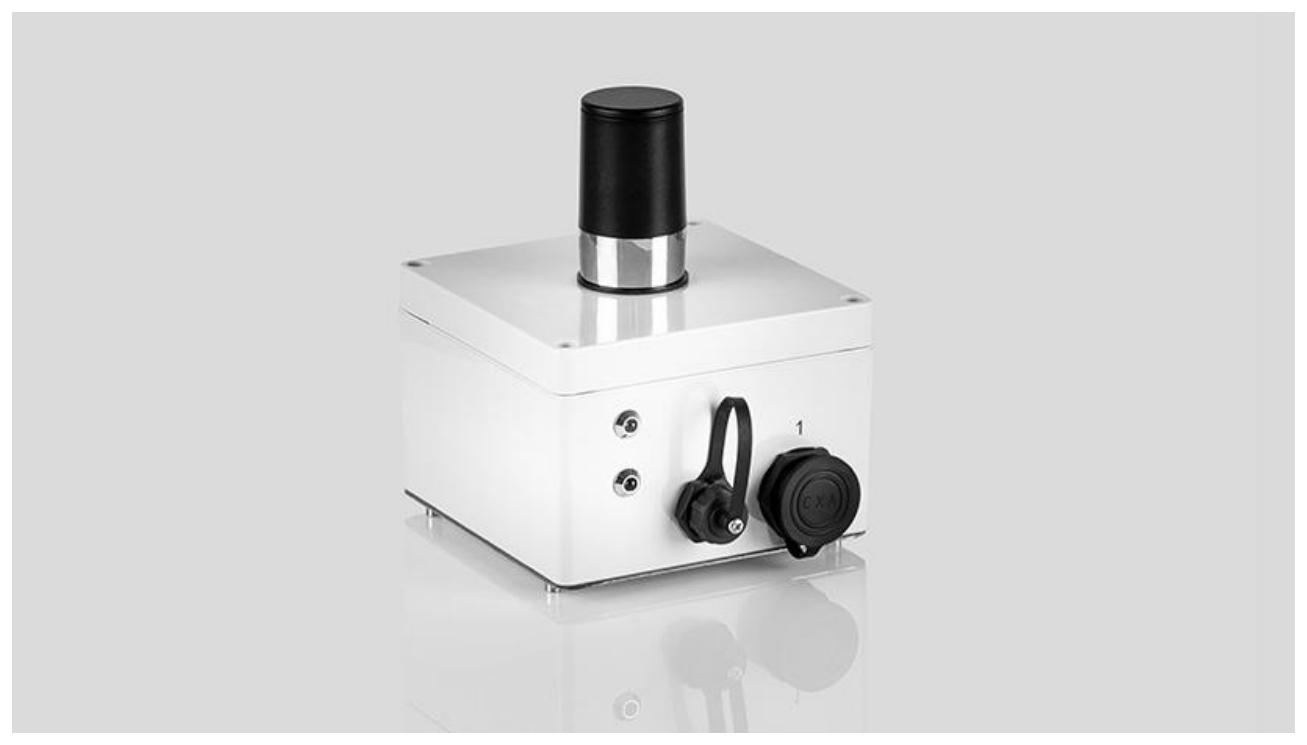

Figure 3 MDT-RTU (Remote Telemetry Unit) 


\subsubsection{VW-RTU}

The Vibrating Wire (VW) RTU is capable of reading up to four vibrating wire sensors with thermistors from all manufacturers, which could include piezometers, pressure cells, stress cells, strain gauges, tilt meters and many others for monitoring a wide range of physical parameters. This is shown in Figure 4. Similar to other RTU, this unit also has on-board storage capacity to store readings in the event of network loss.

\section{Figure 4 VW-RTU (Remote Telemetry Unit)}

\subsubsection{SAA-RTU}

These are capable of reading up to four Shape-Accelerometer-Arrays used for 3D borehole profile monitoring in slope stability applications, tunnel convergence monitoring, and monitoring settlement profiles. An image of the SAA-RTU is shown in Figure 5.

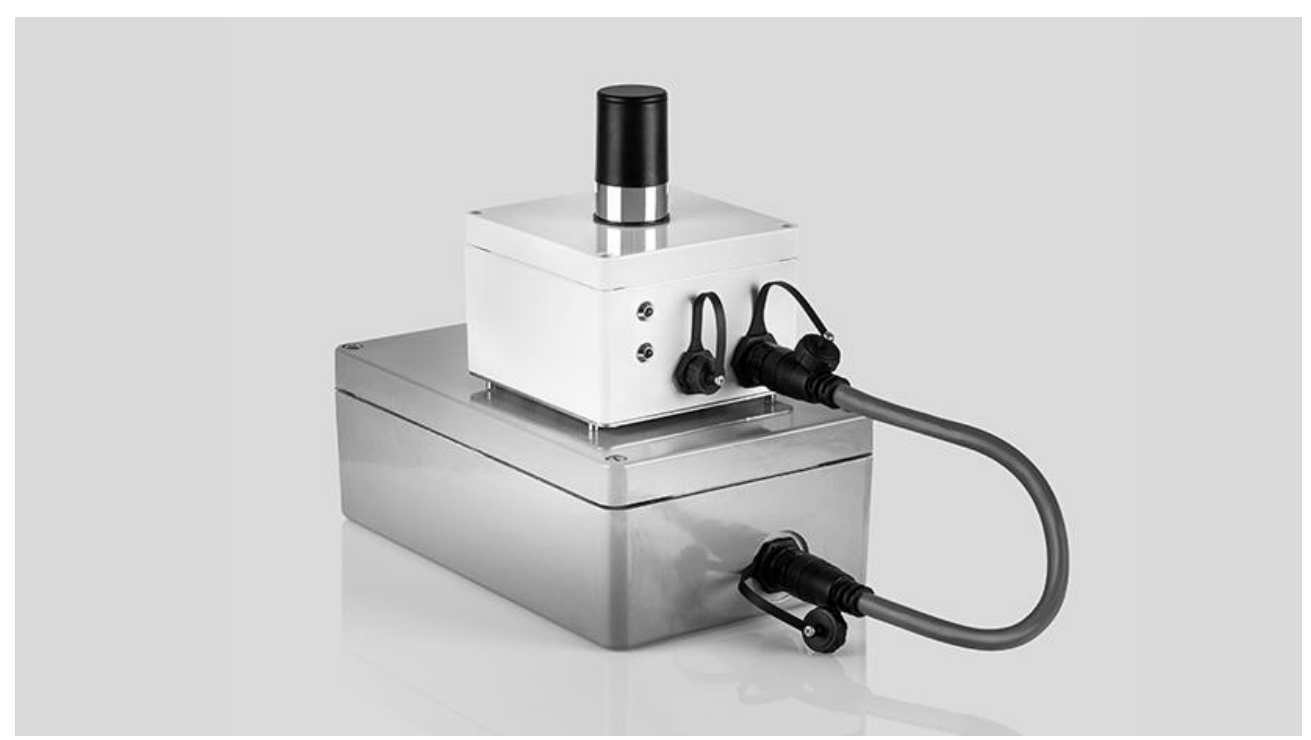

\section{Figure 5 SAA-RTU (Remote Telemetry Unit)}

\section{Benefits of battery-powered wireless technology}

There are a multitude of benefits of using battery-powered technology for wireless sensor networks in a mining environment; one of the most notable is the ease and speed of deployment, and re-deployment if required. This comes from the fact that the installer themselves simply need to range the nodes using the red and green status signal indicators and cable tie them in place for installation, but also the fact that 
electrical and IT departments do not need to be engaged for installing power outlets or configuring network details. The involvement of additional departments can often involve numerous work orders on many sites, creating lengthy delays in the installation process.

This rapid installation is of great benefit to underground mining, as often the areas where installation is planned have limited access due to production activities and have tight timeframes for installation.

The dynamic nature of many underground mines also lends itself to utilising battery-powered wireless data acquisition systems. Whilst many instruments are certainly installed in life of mine areas, in many cases a monitoring program may be deployed in temporary areas such as extensometers around a stope draw point that will only be active for the life of a stope, or monitoring barricade pressures during a backfill. Battery-powered wireless technology allows the system to be simply re-located by cutting cable ties on the nodes, and re-deploying the network to the existing or alternately new gateway location as required.

With no cables to be run, battery-powered systems are also far less likely to be damaged from mine traffic than alternative cabled systems. A common problem with data integrity in a mining environment is damage to lead wires, often caused by Load-Haul-Dump (LHD) loaders or other heavy vehicle traffic. With battery-operated units kept close to the borehole collar or sensor location, cable run length and therefore damage risk can be minimised.

Limitations of this network are that it is a low bandwidth solution, and has been designed this way to achieve the low power consumption targets required. Therefore it is not suitable for high bandwidth data applications such as seismic monitoring, or other high frequency dynamic monitoring.

\section{$4 \quad$ Case studies}

\subsection{Argyle Diamond Mine, Rio Tinto}

During 2014, MDT Australia was engaged to deploy an automated data acquisition system in the extraction level workshop at Argyle Diamond Mine (shown in Figure 6), to collect data from a number of MPBX installed in the walls and back of the workshop for monitoring ground deformation. Key requirements of the system were that it needed to be rapidly deployed due to high traffic in the extraction level, be plug and play with the existing sensors for simple connection, and be battery powered with mains power unavailable at many instrument locations - making the network discussed in this paper a suitable option.

The system was deployed during December 2014 with three days on site, bridging from a network switch at a nearby substation wirelessly to instrument locations up to $100 \mathrm{~m}$ away, with real time data from the installed instruments available immediately upon commissioning. Further work has now been conducted to integrate 27 vibrating wire stress cells and further MPBX into the network, installed as part of a pillar monitoring project, for monitoring stress and deformation in the relevant rock mass.

There are long term plans to integrate over $100 \mathrm{MPBX}$ and instrumented cable bolts into the wireless network, that will be installed throughout the mine's extraction level and crusher chambers. 


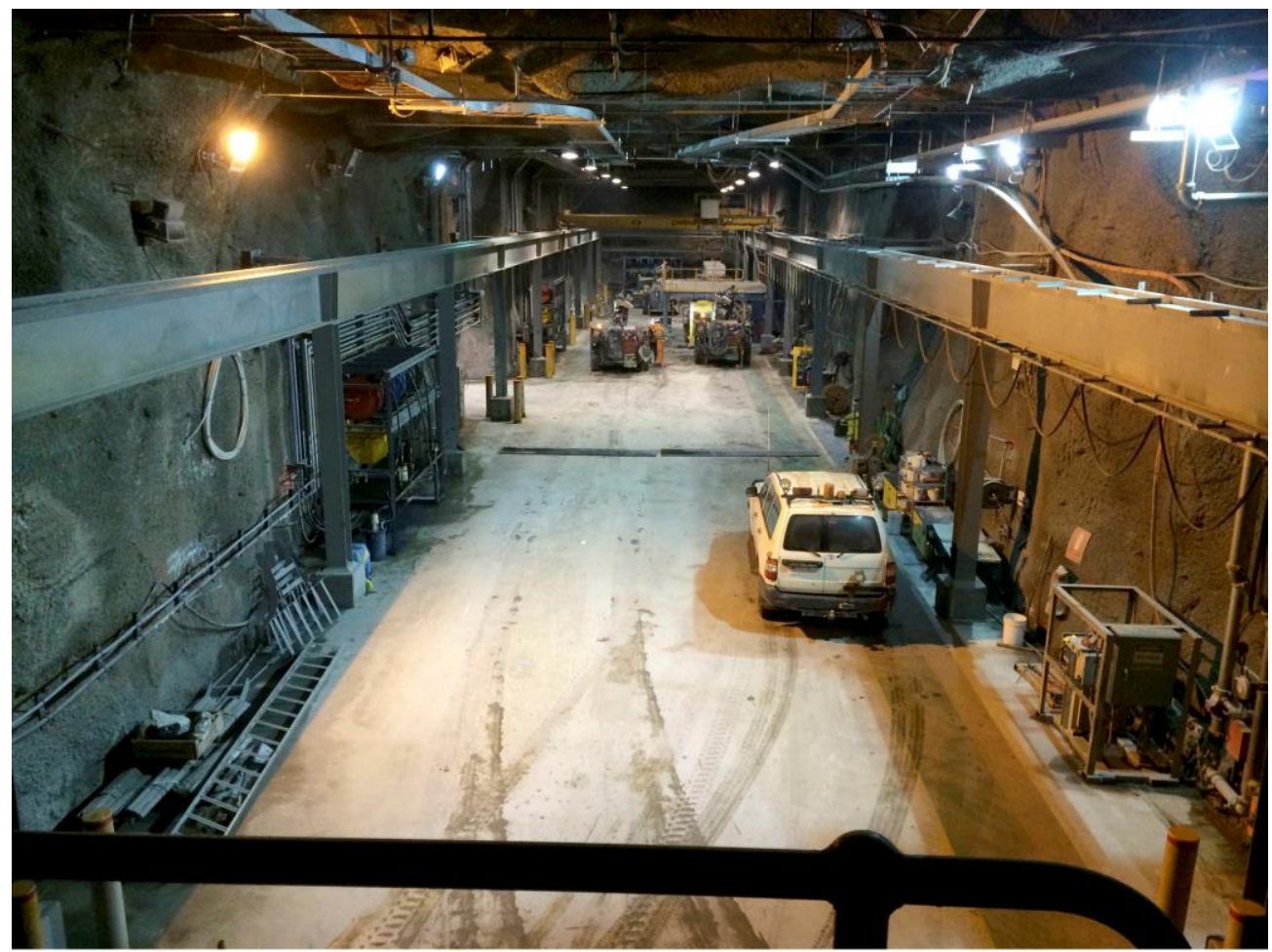

\section{Figure 6 Extraction level workshop}

\subsection{Mount Isa Copper Operations (MICO), Glencore}

MICO has been manually monitoring the integrity of the X41 Shaft, a key life of mine asset at Mount Isa Mines, for over 20 years with a series of faults that run through the shaft profile creating concern for structural damage within the shaft (Bruneau 2000; Bruneau et al. 2003). With several stopes planned for the second half of 2015 onwards bringing mining closer to the shaft boundary than ever previously, the need for a real time monitoring network was necessary to preserve the integrity of the structure as mining advances. MDT Australia in collaboration with pitt\&sherry consultants designed a comprehensive monitoring network to achieve these requirements (Hills 2014), consisting of:

- 48 vibrating wire strain gauges installed in clusters of four on selected shaft buntons.

- 32 vibrating wire tilt meters installed at a number of locations on all four cage guides.

- 36 single point extensometers, installed equally spaced around the circumference at six horizons to monitor deformation in the shaft liner.

- Over five telescoping deformation monitors installed across rockbolts in drives surrounding the shaft to monitor convergence and shear within the drive.

All of which were required to be linked to a near real time data acquisition system for access from surface with email alarm notification functionality.

Key limitations in this project were there was no access to mains power from within the shaft where sensors are to reside, the timeframe for installation work within the shaft was limited due to shaft usage during the day (only a 6-8 hour window each night over night shift), and network connectivity was only available at 19 level approximately $930 \mathrm{~m} \mathrm{BGL}$ and around $100 \mathrm{~m}$ from the shaft plat. Based on these requirements a MineHop battery-powered network was proposed, which would allow sensors at each elevation to be connected over a short distance $(<8 \mathrm{~m})$ to an MDT-RTU or VW-RTU cable tied in place within the shaft ladder way and data relayed to a wireless node below at the shaft plat at 19 level. 
The system was deployed in March 2015 over a 2-week period, including instrumentation and the RF wireless network with assistance from MICO personnel. The network began at 19 level with a gateway connected to the existing seismic system network, and using wireless nodes to link to the shaft plat and wirelessly up the shaft from there to instrument locations. During initial stages of deployment wireless signal was achieved between wireless nodes at 19 level (930 m BGL) and 15 level (715 m BGL), a distance of over $210 \mathrm{~m}$ with reliable data retrieval still available, before further RTU were put in place between the units to read the associated sensors. The complete network consisted of $20 \mathrm{VW}-\mathrm{RTU}, 5 \mathrm{MDT}-\mathrm{RTU}$ and four standard infrastructure nodes spread over a vertical distance of $457 \mathrm{~m}$.

MICO will be collecting baseline data from the system over the coming months before first stope firing begins in around July this year, and have plans to expand the network to collect data from geotechnical instruments in other underground areas as well as from numerous vibrating wire piezometer wells on surface.

\section{$5 \quad$ Upcoming developments}

Amongst many areas of research and development being undertaken to improve this wireless technology, the following are key areas of note.

\subsection{High bandwidth on demand}

As mentioned earlier in this paper, one of the limitations of this battery powered technology is that it is low bandwidth and unsuitable for high bandwidth monitoring applications such as microseismic at this point in time.

Work is currently being undertaken to develop a high bandwidth on demand module, that would enable bursts of high bandwidth data to be transmitted as required for example when monitoring a seismic event. When not required the system would remain in a dormant low bandwidth state to conserve power.

\subsection{Wireless microseismic monitoring}

One of the biggest downfalls of microseismic systems in a dynamic underground environment is the damage to cabling. As a result of the research being conducted for on demand high bandwidth functionality (as discussed above), research is also being undertaken to enable data from microseismic sensors to be transmitted wirelessly instead of through traditional cabled solutions. The objective of this is to reduce cable damage and create a more reliable microseismic network and monitoring program.

In addition to reducing cable damage the research is also focused on integrating microseismic sensors into the toe of a SMART extensometer, which will allow mines to drill one hole to satisfy the requirements of microseismic and traditional geotechnical monitoring, substantially saving on drilling costs.

\section{Acknowledgement}

We would like to acknowledge the Geotechnical Staff at Mount Isa Copper Operations and Argyle Diamond Mine for their support in these projects and allowing us to publish these case studies.

We would also like to thank Peter Hills from pitt\&sherry consultants for his support in the X41 Shaft instrumentation project.

\section{References}

Bruneau, G 2000, 'The influence of faulting on the structural integrity of the X41 Shaft, Copper Mine, Mount Isa, Australia' Masters thesis, University of Laval.

Bruneau, G, Tyler, DB, Hadjigeorgiou, J \& Potvin, Y 2003, 'Influence of faulting on a mine shaft-a case study: part I-Background and Instrumentation', International Journal of Rock Mechanics and Mining Sciences, vol. 40, no. 1, pp. 95-111.

Hills, P 2014, Mount Isa copper operations X41 Shaft recommendations for instrumentation and monitoring, Rev 00, pitt\&sherry. 
